\title{
Artificially structured boundary plasma trap
}

Cite as: Phys. Plasmas 26, 092509 (2019); doi: 10.1063/1.5116349

Submitted: 24 June 2019 - Accepted: 5 September 2019 .

Published Online: 20 September 2019

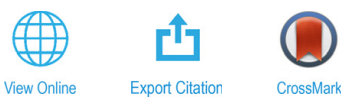

R. M. Hedlof ${ }^{1,2}$ and C. A. Ordonez ${ }^{1, a)}$ (D)

\author{
AFFILIATIONS \\ ${ }^{7}$ Department of Physics, University of North Texas, Denton, Texas 76203, USA \\ ${ }^{2}$ Ball Aerospace, Albuquerque, New Mexico 87106, USA \\ a) Author to whom correspondence should be addressed: cao@unt.edu
}

\begin{abstract}
A computer simulation is presented of single-species non-neutral plasma confinement using an artificially structured boundary. The artificially structured boundary produces a spatially periodic static electromagnetic field along the plasma periphery such that the spatial period of the applied field is much smaller than the dimensions of the confined plasma. The simulated non-neutral plasma self-consistently produces an electrostatic potential energy well for oppositely signed charged particles. The results support the prospect of developing plasma spacecharge based confinement, with an unmagnetized plasma of one species of charged particles confined by an electric field produced by an edge-confined plasma of a second species of charged particles. The Warp particle-in-cell code is used for the simulations.
\end{abstract}

Published under license by AIP Publishing. https://doi.org/10.1063/1.5116349

\section{INTRODUCTION}

In Ref. 1, the existence of plasma equilibria was predicted involving two relaxed plasma species with one species confined by the space charge of the other species. A self-consistent finite-difference numerical approach was used for the study, which developed an understanding of how an edge-confined electron plasma produces a threedimensional electrostatic potential well and how an ion plasma can be confined within a neutral plasma region inside of the well. Equilibria were found for which the magnitude of the difference in an ion's potential energy between the plasma's geometric center and edge was much larger than the ion temperature in energy units. ${ }^{1}$ Along with ions confined by the space charge of electrons, plasma space-charge based confinement is also envisioned for confining antiprotons by the space charge of positrons.

Antihydrogen production for conducting gravity experiments and high precision spectroscopy experiments relies heavily on plasma confinement and manipulation techniques. ${ }^{2-6}$ Penning traps that are nested within neutral atom traps are used by the ALPHA and ATRAP experiments to simultaneously synthesize and confine antihydrogen., ${ }^{2,3}$ However, the strong magnetic field that is employed by Penning traps for radial plasma confinement reduces the three-body recombination rate by roughly an order of magnitude as compared to the rate when there is no magnetic field present. $^{7,8}$ Furthermore, positron space charge and collisions between antiprotons during the mixing process may significantly reduce the number of cold antihydrogen atoms that are suitable for further experimentation., ${ }^{9,10}$
A concept referred to as an artificially structured boundary (ASB) may provide an alternative to the nested Penning trap for combining multispecies plasmas. In the context of the present work, an ASB is an arrangement of electrodes and electromagnets that creates a spatially periodic electromagnetic field, which can be used to confine nonneutral and partially neutralized plasmas. If the dimensions of an ASB plasma trap are chosen such that the spatial period of the electromagnetic field is much smaller than the dimensions of the plasma confinement volume, then the bulk of the plasma will be effectively free of externally applied electromagnetic fields. As envisioned, an ASB trap could be used to confine a positron plasma along its edge, and the space charge of the positron plasma could create an electrostatic potential energy well for confining an antiproton plasma. Such a confinement scheme was investigated in Ref. 1 for electron-ion systems, without considering the structure of an electron-reflecting boundary.

Several configurations of electrodes and electromagnets (or permanent magnets) can be envisioned that can be considered as ASBs. For example, a planar geometry could be constructed by arranging a large number of magnetic dipoles in a grid pattern with the dipole moment of adjacent magnets directed in opposite directions. A conceptually simpler alternative, referred to as a "picket-fence," can be constructed using a sequence of equally spaced long wires, with adjacent wires carrying current in opposite directions. Charged particle reflection from the magnetic field of the picket-fence configuration was investigated in Ref. 11, and single-particle trajectory simulations presented in Ref. 12 indicate that it may be possible to reflect effectively all incident individual particles from the picket-fence ASB with 
the addition of a suitable applied electrostatic field. Other work involving charged particle reflection from ASBs has been reported elsewhere, ${ }^{13-16}$ and various confinement schemes utilizing electrostatically plugged magnetic cusps have been discussed in the context of fusion related applications in Ref. 17.

In Ref. 12, a classical trajectory Monte Carlo study of single particle trajectories was used to find optimal relative sizes of plasma-facing electrodes. In the work reported here, a particle-in-cell code is used for conducting a self-consistent study, regarding whether a cylindrically symmetric ASB enclosure can confine a non-neutral plasma using the optimized electrode relative sizes found in Ref. 12. The Warp particlein-cell code is used for the work reported here. ${ }^{18}$ An initial study with Warp regarding plasma confinement using a cylindrically symmetric ASB was reported previously, ${ }^{19}$ but only radial confinement was considered (not axial confinement) in Ref. 19. Section II describes the ASB trap model and the externally controlled parameter values selected for a simulation of plasma confinement within the trap. Section III describes the computer simulation of plasma confinement within the ASB trap. Section IV presents the results obtained. A discussion and concluding remarks are provided in Sec. $\mathrm{V}$.

\section{TRAP PHYSICAL MODEL AND EXTERNALLY CONTROLLED PARAMETER VALUES}

Plasma confinement within a trap that employs a cylindrically symmetric picket-fence ASB is investigated via computer simulation. A cross-sectional view of the ASB trap is shown in Fig. 1. The trap is cylindrically symmetric about the $z$-axis and consists of a series of electrodes and electromagnetic coils that are arranged in such a way so as to produce a three-dimensional confinement volume for either a positively or negatively charged plasma species. In what follows, positive voltages are applied to "plugging" electrodes (gray rectangles in Fig. 1) in order to confine a positron plasma, and all electrodes that are white in Fig. 1 are held at ground potential $(0 \mathrm{~V})$.

The trap may be thought of as having three sets of components, where each set of components contributes to plasma confinement in a specific way. The first set of components produces two electrostatic potential barriers along the axis of symmetry. The axial-barrier components consist of four cylindrical electrodes, each of inner radius $R_{g}=0.5 \mathrm{~cm}$ and axial length $L_{g}=0.25 \mathrm{~cm}$, which are coaxial with the $z$-axis, with two electrodes located on each end of the trap. The outermost electrodes (dark gray) are biased to a positive potential of $V_{g}=50 \mathrm{~V}$, while the inner two electrodes are held at $0 \mathrm{~V}$. An axial magnetic field is present along the axis of symmetry inside of the axial-barrier components. Loading the trap is envisioned to occur experimentally through axial-barrier components joined to Penning traps, but the presence of Penning traps is not considered in the present work.

The second set of components, hereafter referred to as end caps, provides off-axis confinement for the positron plasma in the $z$-direction. Each of the two end caps consists of a sequence of concentric cylindrical electrodes and circular electromagnetic coils. Adjacent electromagnetic coils carry current in opposite directions and are spaced equidistant from one another in the radial direction. The innermost electromagnetic coil of each end cap has a radius of $R_{g}=0.5 \mathrm{~cm}$ and is radially flush with the surface of the grounded electrode of the corresponding axial-barrier component. Each end cap creates a cylindrically symmetric picket-fence magnetic field in the radial direction that is

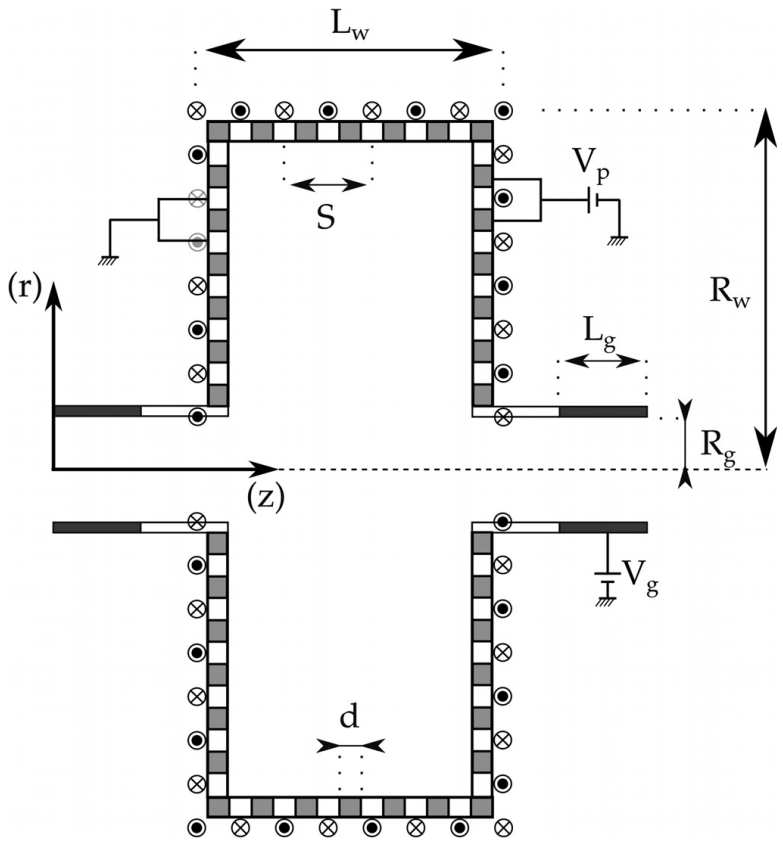

FIG. 1. Illustration showing a cross-sectional view of the ASB trap. Electrodes are depicted as gray or white rectangles, while electromagnetic coils are depicted as circles. The direction of current flow in each electromagnet is indicated by the " $\times$ " or "dot" inside of the circles. All components of the trap are azimuthally symmetric about the $z$-axis. The figure is not to scale, and the figure qualitatively represents case $\mathrm{L} 1$ defined in Table I.

plugged electrostatically as was done for the planar and cylindrical arrangements presented in Refs. 12 and 19, respectively. The third set of components of the trap provides radial confinement of the positron plasma and is hereafter referred to as the trap wall. The trap wall considered here is similar to that which was investigated in Ref. 19 and produces an electrostatically plugged, cylindrically symmetric, picketfence magnetic field. The electromagnets in the trap wall have a radius of $R_{w}$, and the distance in the $z$ dimension between geometric centers of electromagnetic coils in each of the end caps is denoted $L_{w}$. The parameters $R_{w}$ and $L_{w}$ have values that are varied in the work presented here. The plugging electrodes for both the trap wall and the end caps are all biased to a voltage of $V_{p}=24 \mathrm{~V}$, and each of the circular electromagnetic coils carries a single-turn current of $750 \mathrm{~A}$.

Each electromagnetic coil is approximated as an infinitesimally thin current-carrying circular loop. The separation distance of adjacent electromagnetic coils in the end caps and the trap wall is chosen such that the spatial period of the magnetic field is $S=1.0 \mathrm{~cm}$ for both components. The locations of the plugging electrodes for the end caps and trap wall are chosen such that each is centered midway between adjacent electromagnetic coils. The plugging electrodes in the end caps have a radial thickness of $d$, while the plugging electrodes located in the trap wall have an axial length of $d$, where the value, $d=0.4 \mathrm{~S}$ $=0.4 \mathrm{~cm}$, is chosen in accordance with the optimization results reported in Ref. 12. The electromagnets in the end caps and the trap wall are recessed from the available particle confinement volume in 
the radial and axial directions, respectively, by being located a distance of $\delta=0.05 \mathrm{~cm}$ from the surfaces of the electrodes. The available particle confinement volume, excluding the volume inside of the axialbarrier components, is cylindrical with length $L_{w}-2 \delta$ and radius $R_{w}-\delta$. The $z$-coordinates of the electromagnetic coils in the left end cap and right end cap are $z_{l c}=2 L_{g}-\delta$ and $z_{r c}=z_{l c}+L_{w}$, respectively. (Note that the coordinate origin is located at one end of the trap.) The total axial length of the trap, including the axial-barrier components, is $z_{\max }=2 z_{l c}+L_{w}$.

\section{SIMULATION DETAILS}

The total magnetic field inside of the trap $\boldsymbol{B}(r, z)$ is the superposition of the magnetic fields produced by the left and right end caps, $\boldsymbol{B}_{l c}(r, z)$ and $\boldsymbol{B}_{r c}(r, z)$, respectively, and the magnetic field produced by the trap wall $\boldsymbol{B}_{w}(r, z)$,

$$
\boldsymbol{B}(r, z)=\boldsymbol{B}_{l c}(r, z)+\boldsymbol{B}_{r c}(r, z)+\boldsymbol{B}_{w}(r, z) .
$$

Here,

$$
\boldsymbol{B}_{l c}(r, z)=\sum_{i=0}^{N_{c}-2}(-1)^{i} \boldsymbol{B}_{s}\left(r, z-z_{l c}, a_{i}\right)
$$

and

$$
\boldsymbol{B}_{r c}(r, z)=\sum_{i=0}^{N_{c}-2}(-1)^{i+1} \boldsymbol{B}_{s}\left(r, z-z_{r c}, a_{i}\right),
$$

where $\boldsymbol{B}_{s}(r, z, a)$ is the magnetic field produced by a single current loop of circular radius $a$ and $a_{i}=R_{g}+i S / 2$ is the radius of the $i^{\text {th }}$ electromagnetic coil. The magnetic fields of $N_{c}-1$ electromagnetic coils are superposed for each end cap by Eqs. (2) and (3). The magnetic fields produced by two end-cap coils with radius $R_{w}$ $=R_{g}+\left(N_{c}-1\right) S / 2$ which are also part of the trap wall are excluded in Eqs. (2) and (3), and $N_{c}$ is the number of electromagnetic coils in each end cap. The magnetic field produced by a current loop is given, for example, by Ref. 20, and $\boldsymbol{B}_{s}(r, z, a)$ represents the magnetic field produced at radial and axial coordinates $(\mathrm{r}, \mathrm{z})$ by a circular current loop that is centered at the origin of the cylindrical coordinate system and that has an axis of symmetry aligned with the $z$ axis. The magnetic field produced by the trap wall is

$$
\boldsymbol{B}_{w}(r, z)=\sum_{i=0}^{N_{w}-1}(-1)^{i+N_{c}+1} \boldsymbol{B}_{s}\left(r, z-z_{i}, R_{w}\right)
$$

where the $z$-coordinate of the $i$ th electromagnetic coil is $z_{i}=z_{l c}$ $+i S / 2$ and $N_{w}$ is the number of electromagnetic coils making up the wall, including the two shared with the end caps that are separated axially by the distance, $L_{w}=\left(N_{w}-1\right) S / 2$. The values of $R_{w}$ and $L_{w}$ are restricted such that $N_{w}$ and $N_{c}$ are even numbers.

Simulations are conducted using a two-dimensional electrostatic version of the Warp code ${ }^{18}$ where the electrostatic potential due to the trap and plasma is calculated self-consistently at radial and axial cylindrical coordinates at each time step, and the magnetic field produced by the plasma is neglected. The assumption of azimuthal symmetry allows three-dimensional potential profiles to be self-consistently determined with a two-dimensional grid on the $(r, z)$ plane. The same grid spacing is used in radial and axial directions, $\Delta r=\Delta z=\Delta s$. Here, $\Delta s$ is chosen to be the smaller of two values according to

$$
\Delta s=\min \left(\frac{\lambda_{D e}}{4}, \frac{S}{N_{s}}\right),
$$

where $\lambda_{D e}=\sqrt{\epsilon_{0} k_{B} T_{0 e} /\left(n_{0 e} e^{2}\right)}$ is the Debye length of a positron plasma with density $n_{0 e}$ and temperature $T_{0 e}, \epsilon_{0}$ is the permittivity of free space, $k_{B}$ is Boltzmann's constant, and $e$ is the charge of a positron. $T_{0 e}$ is the temperature of positrons while being loaded into the trap, and $n_{0 e}$ is the initial density of positrons as defined below. $N_{s}$ is a minimum number of grid points per spatial period of the magnetic field used in each simulation. For all simulations in the present work, a grid resolution of $N_{s} \geq 90$ is used. For each simulation, Dirichlet boundary conditions are imposed on the electrostatic potential $\phi(r, z)$ to model an electrode configuration similar to that shown in Fig. 1 with varying values of $R_{w}$ and $L_{w}$, while Neumann boundary conditions are imposed on the electrostatic potential at $z=0$ and $z=z_{\max }$ such that

$$
\left[\frac{\partial \phi(r, z)}{\partial z}\right]_{z=0}=\left[\frac{\partial \phi(r, z)}{\partial z}\right]_{z=z_{\max }}=0 .
$$

The electrostatic potential and magnetic field are defined on the computational grid, and the forces acting on the macroparticles off the grid are computed via interpolation.

Positron plasma macroparticles are loaded into a cylindrically symmetric uniform injection volume over the course of the first $0.5 \mu \mathrm{s}$ of each simulation. The plasma is allowed to evolve self-consistently, while the positron macroparticles are being injected during $0.5 \mu \mathrm{s}$, and the simulation continues for another $19.5 \mu$ s thereafter. Thus, each simulated evolution of the system lasts $20 \mu$ s. Each macroparticle in the system represents 50 plasma particles (i.e., the assigned macroparticle weight is 50). The injection volume is chosen to be a cylinder of radius, $R_{i n j}=R_{w}-\sqrt{2} S$, and length, $L_{i n j}=z_{i n j, \max }-z_{i n j, \min }$, where $z_{i n j, \max }=z_{r c}-S$ and $z_{i n j, \min }=z_{l c}+S$ are the maximum and minimum $z$-coordinates of the injection volume, respectively. The initial velocity components $v_{0 x}, v_{0 y}$, and $v_{0 z}$ of each macroparticle are sampled from a three-dimensional nondrifting Maxwellian velocity distribution associated with a temperature of $T_{0 e}=40 \mathrm{~K}$. The total number of macroparticles $N_{e}$ in each simulation is chosen such that the density of the plasma would be $n_{0 e}=10^{6} \mathrm{~cm}^{-3}$ had all plasma particles been initialized within the injection volume instantaneously at time $t=0 \mathrm{~s}$.

Using the two-dimensional electrostatic version of the Warp code, plasma particles are allowed to move in three-dimensions according to the ASB applied magnetic field and the self-consistently calculated electric field. For all simulations in the work presented here, the Boris algorithm is used to push particles with a time step of $\Delta t$ $=5.6 \mathrm{ps}$. The value of $\Delta t$ is chosen according to $\Delta t=0.1 / \omega_{c}$, where $\omega_{c}$ is the angular frequency of the cyclotron motion of a positron moving in a $B_{\max }=0.1014 \mathrm{~T}$ magnetic field. Here, $B_{\max }$ is the magnitude of the magnetic field at a point that is very close to the radius of one of the electromagnetic coils in the trap wall. If the trajectory of a particle intersects the $z=0.0 \mathrm{~cm}$ or $z=z_{\max }$ planes, or any of the trap electrodes, the particle is considered lost and is removed from the simulation. After each simulation is complete, the position and momentum of each particle that remains in the trap and the electrostatic potential $\phi(r, z)$ are recorded.

It is illustrative to consider some length scales. A positron with mass $m$ traveling in a straight line during $20 \mu$ s at the injection thermal speed, $\sqrt{k_{B} T_{0 e} / m}$, would travel $49 \mathrm{~cm}$. The Debye length for the injection parameter values chosen is $\lambda_{D e}=0.44 \mathrm{~mm}$. A positron traveling 
TABLE I. Parameter values used in the ASB trap simulations and some results. The total number of injected macroparticles $N_{e}$ is chosen such that the positron density (with 50 positrons per macroparticle) would have initially been $n_{0 e}=10^{6} \mathrm{~cm}^{-3}$ inside of the injection volume had injection occurred instantaneously at $t=0 \mathrm{~s}$.

\begin{tabular}{lrcccc}
\hline \hline Case no. & $L_{w}(\mathrm{~cm})$ & $R_{w}(\mathrm{~cm})$ & $N_{e}$ & $\Delta \phi(\mathrm{V})$ & Particles lost $(\%)$ \\
\hline L1 & 3.5 & 4.0 & 565634 & 0.21 & 0.0 \\
L2 & 4.5 & 4.0 & 969690 & 0.52 & 0.0 \\
L3 & 5.5 & 4.0 & 1373725 & 0.77 & 0.0 \\
L4 & 6.5 & 4.0 & 1777741 & 0.91 & 0.0 \\
L5 & 7.5 & 4.0 & 2181771 & 1.09 & 0.0 \\
L6 & 8.5 & 4.0 & 2585792 & 1.16 & 0.0 \\
L7 & 9.5 & 4.0 & 2989807 & 1.24 & 0.0 \\
L8 & 10.5 & 4.0 & 3393912 & 1.34 & 0.0 \\
L9 & 11.5 & 4.0 & 3797913 & 1.33 & 0.0 \\
L10 & 12.5 & 4.0 & 4201863 & 1.36 & 0.0 \\
L11 & 13.5 & 4.0 & 4605889 & 1.38 & 0.0 \\
R1 & 8.5 & 3.0 & 948470 & 0.56 & 0.0 \\
R2 & 8.5 & 4.0 & 2585792 & 1.16 & 0.0 \\
R3 & 8.5 & 5.0 & 5027279 & 1.74 & 0.0 \\
R4 & 8.5 & 6.0 & 8273063 & 2.20 & 0.0 \\
R5 & 8.5 & 7.0 & 12323097 & 2.62 & 0.0 \\
R6 & 8.5 & 8.0 & 17177378 & 2.99 & $1.2 \times 10^{-5}$ \\
R7 & 8.5 & 9.0 & 22836240 & 3.32 & 0.11 \\
\hline \hline
\end{tabular}

perpendicular to a uniform magnetic field of strength $B_{\max }$ would have a thermal gyroradius $\sqrt{m k_{B} T_{0 e} /\left(e^{2} B_{\max ^{2}}\right)}$ of $1.4 \mu \mathrm{m}$.

\section{RESULTS}

A parametric study is conducted in which the length and radius of the trap wall are varied. Table I shows the parameter values used and gives some of the results. For case numbers beginning with $\mathrm{L}$, the radius of the trap is held constant with $R_{w}=4.0 \mathrm{~cm}$, while the length of the trap wall is varied with $3.5 \mathrm{~cm} \leq L_{w} \leq 13.5 \mathrm{~cm}$. For case numbers beginning with $\mathrm{R}$, the length of the trap wall is held constant with $L_{w}=8.5 \mathrm{~cm}$, while the radius of the trap is varied with $3.0 \mathrm{~cm}$ $\leq R_{w} \leq 9.0 \mathrm{~cm}$. For each simulation, a non-neutral positron plasma is injected as was described in Sec. III. In each of the simulations where the length of the trap is varied, no positrons are lost. Likewise, no particles are lost for cases R1-R5. However, for cases R6 and R7, $1.2 \times 10^{-5} \%$ ( 2 macroparticles) and $0.11 \%$ (25 621 macroparticles) of the initial positron population are lost, respectively. The losses are attributed to space charge effects, as discussed below.

\section{A. Electrostatic potential}

Figure 2 shows the electric potential $\phi(r, z)$ in the trap at the end of a simulation, at time $t=20 \mu \mathrm{s}$, for case R7. The electric potential typically has an extremum close to the geometric center of the trap located on the $z$ axis. The electric potential also has saddle points, each of which occurs within a magnetic cusp in front of and approximately centered with a plugging electrode. A positron at a saddle point experiences an increase in potential energy if it moves along the local magnetic field line toward or away from the associated plugging electrode. The minimum height of the electrostatic potential hill formed by the

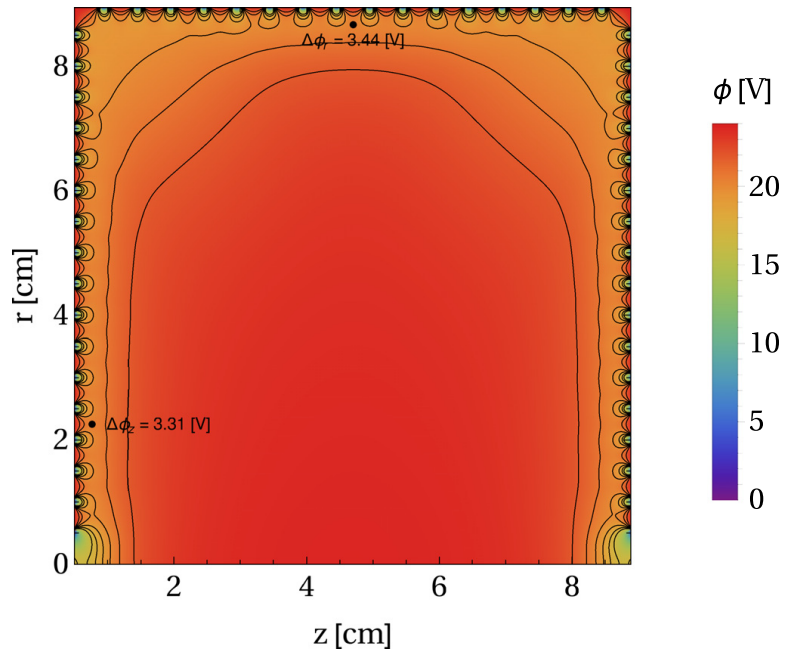

FIG. 2. Electrostatic potential in the trap at time $t=20 \mu$ s for case R7. The black dots indicate the locations of the saddle points where $\Delta \phi_{r}$ and $\Delta \phi_{z}$ are evaluated.

space charge of the positron plasma is defined as the difference $\Delta \phi$ $=\phi_{c}-\phi_{s, \text { max }}$ between the potential at the center of the trap $\phi_{c}$ and the maximum value of the electrostatic potential $\phi_{s, \max }$ at any one of the saddle points. The value of $\Delta \phi$ is first evaluated separately for saddle points along the trap wall $\left(\Delta \phi_{r}\right)$ and saddle points along the end caps $\left(\Delta \phi_{z}\right)$, and the overall minimum hill height is then determined as the smaller of the two values, $\Delta \phi=\min \left(\Delta \phi_{r}, \Delta \phi_{z}\right)$. Figure 2 shows the locations of the saddle points associated with evaluations of $\Delta \phi_{r}$ and $\Delta \phi_{z}$ and the values of $\Delta \phi_{r}$ and $\Delta \phi_{z}$ for the case shown.

In contrast to a positron, an oppositely charged particle at a saddle point experiences a decrease in potential energy if it moves along the local magnetic field line toward or away from the associated plugging electrode. For plasma space-charge based confinement, for example, with antiprotons confined by using the space charge of a positron plasma, the difference in potential between each saddle point and the center of the trap can be determined. The smallest difference in potential would provide the potential energy well depth $e \Delta \phi$ for a confined antiproton. However, each saddle point occurs within a magnetized region. If it is desirable for antiprotons to be confined while being effectively unmagnetized, a potential energy well depth smaller than $e \Delta \phi$ would have to be considered.

Figure 3 shows the height of the space-charge hill in the radial $\Delta \phi_{r}$ and axial $\Delta \phi_{z}$ directions for all of the cases considered. For cases L1-L11, the radial hill height $\Delta \phi_{r}$ is slightly smaller than the axial hill height $\Delta \phi_{z}$ in all simulations. In cases R1 and R2, $\Delta \phi_{r}<\Delta \phi_{z}$, while for cases R3-R7, $\Delta \phi_{r}>\Delta \phi_{z}$. Relatively close values occur for $\Delta \phi_{r}$ and $\Delta \phi_{z}$, indicating that the radial and axial space-charge based confinement properties of the trap may be similar. Also, as either the radius or length of the trap increases, the height of the space-charge hill increases in both the radial and axial directions.

\section{B. Plasma density}

Figure 4 shows the positron density $n_{e}(r, z)$ in the trap at the end of a simulation, at time $t=20 \mu \mathrm{s}$, for case R7. The black dots in Fig. 4 

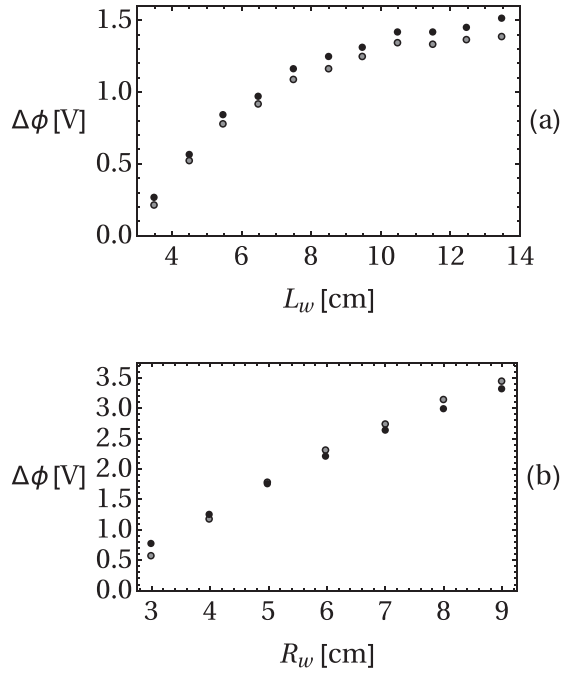

FIG. 3. Radial $\Delta \phi_{r}$ (gray) and axial $\Delta \phi_{z}$ (black) space-charge hill height at time $t=20 \mu \mathrm{s}$ as a function of (a) $L_{w}$ when $R_{w}=4.0 \mathrm{~cm}$ and (b) $R_{w}$ when $L_{w}=8.5 \mathrm{~cm}$.

represent the locations of a sample of 5000 randomly selected particles. The distribution of black dots in Fig. 4 serves to elucidate the particle confinement region that is imposed by the magnetic field, with the distance into a magnetic cusp that particles travel limited by the electric field produced by the electrodes. The particle density variations are represented by the color variations in Fig. 4. For all of the cases, the density near the center of the trap is much smaller than the density near the plasma's edge. Also, a peak in density occurs along the axis of symmetry near the axial barriers.

Figure 5 shows the average central density $n_{c}$ of the positron plasma for all of the cases considered. The average central density is

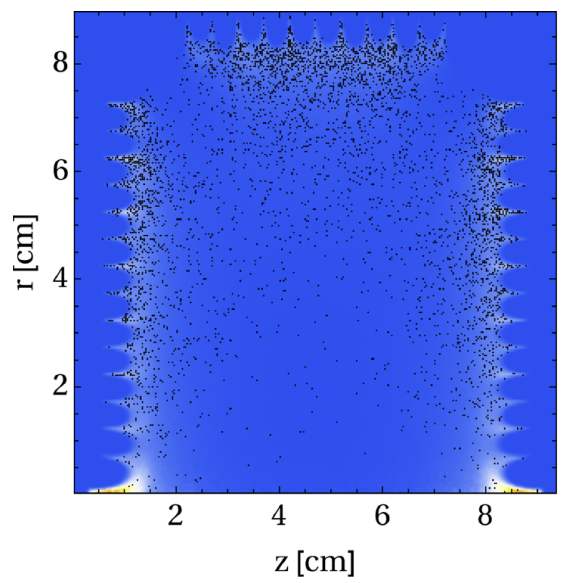

$n_{e^{+}}\left[10^{6} \mathrm{~cm}^{-3}\right]$

FIG. 4. Density of the positron plasma at time $t=20 \mu$ s for case R7. The black dots represent the locations of a small sample of trapped particles.
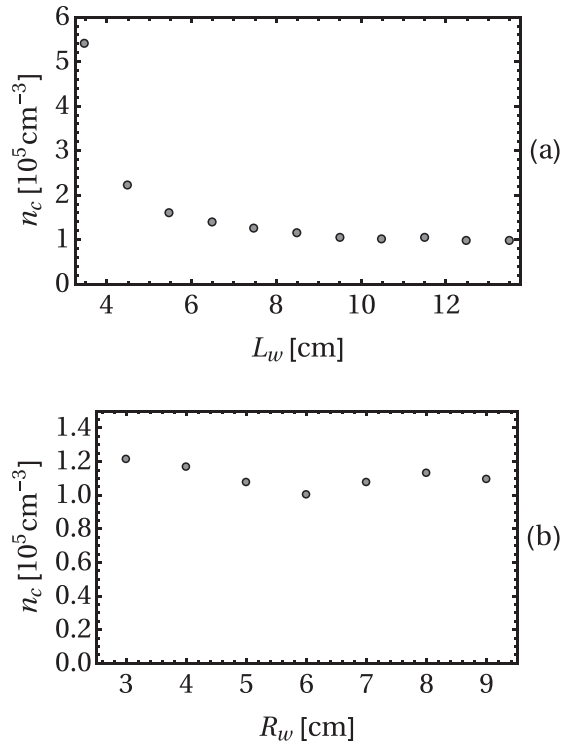

FIG. 5. Average density near the trap's center for (a) cases L1-L11 with $R_{w}=4.0$ $\mathrm{cm}$ and (b) cases R1-R7 with $L_{w}=8.5 \mathrm{~cm}$.

obtained within a cylindrical volume of radius $0.5 \mathrm{~cm}$ and length $1.0 \mathrm{~cm}$ that is coaxial with and centered within the trap. The average central density is relatively unaffected by changes made to the trap length and radius, except that larger values of $n_{c}$ occur for smaller values of $L_{w}$. The latter increase is attributed to an axial confinement volume that approaches $1.0 \mathrm{~cm}$ in length such that the higher density near the plasma edges contribute more to the evaluation of $n_{c}$.

\section{Plasma temperature}

Figure 6 shows the positron temperature $T_{e}(r, z)$ in the trap at the end of a simulation, at time $t=20 \mu \mathrm{s}$, for case R7. Temperature in energy units is evaluated as the average of three variances associated with three Cartesian velocity components, respectively, multiplied by the positron mass. For all of the cases, the temperature near the plasma's edge tends to be larger than near the center of the trap. The temperature increase away from the trap's center is attributed to fueling the plasma about the trap's center in a region of relatively high potential energy. The effects of binary collisions are not included in the simulation, and such collisions may be expected to gradually reduce the spatial temperature variation.

The average central temperature $T_{c}$ is shown in Fig. 7. The average central temperature $T_{c}$ is obtained within a cylindrical volume of radius $0.5 \mathrm{~cm}$ and length $1.0 \mathrm{~cm}$ that is coaxial with and centered within the trap. While the temperature near the edges of the trap is greater than $100 \mathrm{~K}$ for cases L1-L11, the temperature near the trap's center is relatively constant as the length of the trap is varied, being between 49.1 and $57.0 \mathrm{~K}$ for all cases except L1. In contrast, as the radius of the trap increases, with $R_{w}=3.0 \mathrm{~cm}$ in case R1 and $R_{w}=9.0 \mathrm{~cm}$ in case R7, the average central temperature increases from $T_{c}=44.8 \mathrm{~K}$ in case $\mathrm{R} 1$ to $T_{c}=120.1 \mathrm{~K}$ in case R7. The relatively small decrease in $T_{c}$ for 


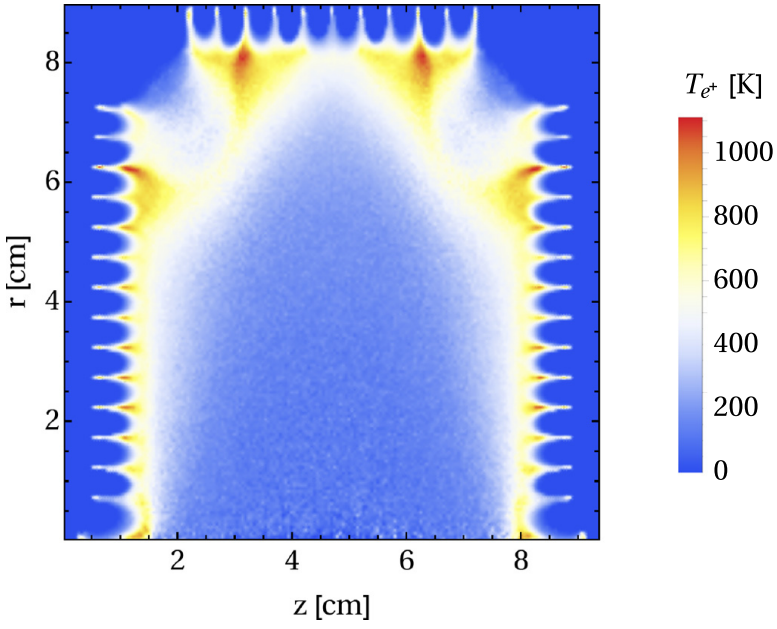

FIG. 6. Temperature of the positron plasma at time $t=20 \mu$ s for case R7.

case R7 relative to case R6 is attributed to a $0.11 \%$ loss of particles in case R7.

\section{DISCUSSION AND CONCLUDING REMARKS}

As indicated in Table I, for all but cases R6 and R7, the number of particles lost from confinement is zero. The effects of space charge can be expected to be the strongest for cases R6 and R7 as a result of having the highest values of $\Delta \phi$, with R7 having the largest value overall. In case R7, the electrostatic potential at the trap's center has a value,
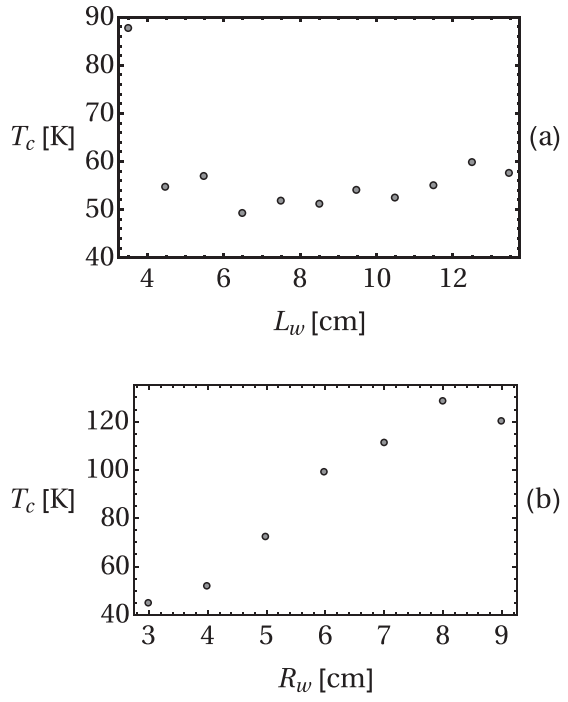

FIG. 7. Temperature near the trap's center for (a) cases L1-L11 when $R_{W}=4.0$ $\mathrm{cm}$ and (b) cases R1-R7 when $L_{w}=8.5 \mathrm{~cm}$. $\phi_{c}=23.84 \mathrm{~V}$, which approaches the electric potential applied to the plugging electrodes, $V_{p}=24 \mathrm{~V}$. Since $\phi_{c} \approx V_{p}$, some positrons born with high potential energy are able to escape confinement. Figure 8 shows the rate at which particles are lost $\dot{N}$ between the time particle injection stops at $t=0.5 \mu \mathrm{s}$ and the end of the simulation at $t=20 \mu \mathrm{s}$. (Note that adjacent small tick marks along the horizontal axis represents a time interval of $1 \mu \mathrm{s}$, and the plotted line begins at $t=0.5 \mu \mathrm{s}$.) Just after the particle injection period ends at $t=0.5 \mu \mathrm{s}$, the loss rate is $\dot{N}=3.73 \times 10^{3} \mu \mathrm{s}^{-1}$. Between $t=0.5 \mu \mathrm{s}$ and $t=1.0 \mu \mathrm{s}$, the loss rate decreases rapidly to $\dot{N}=1.35 \times 10^{3} \mu \mathrm{s}^{-1}$. Particles continue to be lost throughout the duration of the simulation, and the loss rate decreases to $\dot{N}=1.18 \times 10^{3} \mu \mathrm{s}^{-1}$ by $t=20.0 \mu$ s. It is hypothesized that, for times $t>20 \mu \mathrm{s}$, as particles are lost from the trap, a number of effects will occur. The electrostatic potential near the trap's center will decrease, and $\dot{N}$ will approach zero. Also, the plasma will cool evaporatively because particles that escape confinement by reaching the plugging electrodes must have a high enough energy to overcome the electrostatic potential barrier created by the plugging electrodes. However, evolving the system to check the hypothesis was not computationally manageable.

The results of Fig. 3 indicate that as the size of the trap increases, the height of the space-charge hill increases (provided that particles are injected into the trap as described with $n_{0 e}=10^{6} \mathrm{~cm}^{-3}$ ). Such results are in qualitative agreement with the results of Ref. 1, which considered non-neutral and partially neutralized plasma confinement, assuming that a smooth particle-reflecting boundary was present. Additionally, the height of the electrostatic potential hill in case R3 is in quantitative agreement with the result found in case I of Ref. 19. In Ref. 19, particle-in-cell simulations were used to study radial confinement (but not axial confinement) of non-neutral and partially neutralized plasmas in a cylindrically symmetric ASB trap. The work presented here indicates that the radial and axial space-charge based confinement properties of the trap may be similar. Thus, a more comprehensive parametric study, including the presence of an oppositely signed plasma species, may be computationally manageable by using smaller scale simulations, such as those presented in Ref. 19. Such smaller scale simulations may serve to study, for example, density limits in larger-sized (relative to the magnetic field's spatial period) ASB traps that provide plasma space-charge based confinement of a second species.

A recent discussion of numerical heating in particle-in-cell simulations (e.g., with the Boris algorithm) in magnetized plasmas is found

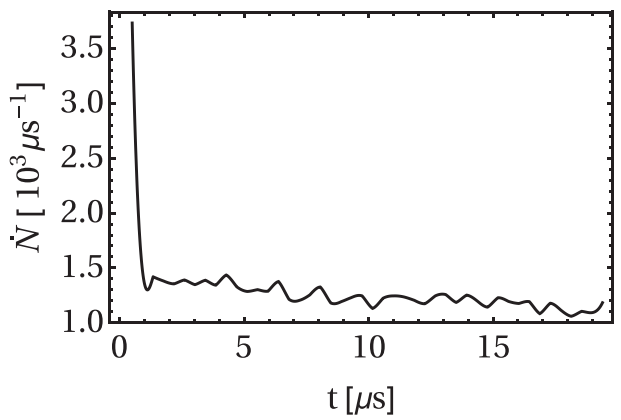

FIG. 8. Rate $\dot{N}$ at which particles are lost for case R7. 
in Ref. 21. According to Ref. 21: (1) "To conserve the energy in the PIC simulation, it is typically advised to use $\omega \Delta t \leq 0.1 \ldots$ " "where $\omega$ is the largest characteristic frequency in the system..." (2) "It is generally accepted that not resolving the electron gyroradius on the spatial grid (when $r_{L}<\Delta x$ ) does not have consequences on the numerical stability due to the homogenous distribution of the particles and that the accurate resolving the gyration by temporal spacing is sufficient." (3) "... for unresolved Larmor radius the electrons are being heated in the direction perpendicular to the magnetic field. These effects can be only partially diminished, but cannot be avoided by using higher-order weighting functions." In the simulations presented here, the positron cyclotron period and gyroradius associated with $B_{\max }$ are expected to be the smallest characteristic time scale and spatial scale, respectively. Although particles that reach a location where the magnetic field strength approaches $B_{\max }$ are likely to be lost, the effects of the transitions between being effectively unmagnetized and being strongly magnetized are not known. For example, it cannot be ruled out that numerical heating and trajectory errors have not had an effect, such as to increase the plasma temperature at the edge or to cause particle losses in cases R6 and R7. However, it would not be expected that numerical heating or trajectory errors would improve particle confinement, and the particle loss results in cases L1-L11 and R1-R5 are expected to remain unchanged even if numerical heating and trajectory errors are reduced.

In summary, particle-in-cell simulations have been presented of radial and axial confinement of a non-neutral plasma within a cylindrically symmetric ASB trap. A parametric study was done by varying the trap's radius and length. No losses of plasma particles occurred for some of the conditions simulated. Losses that did occur were attributed to the effect of space charge because the electric potential at the top of the electrostatic potential hill produced by the positron plasma's space charge was close in value to the electric potential of the plugging electrodes. Plots for the electrostatic potential, plasma density, and plasma temperature were presented for various cases. In the collisionless limit, and for the particle injection method used, the simulations indicate that when non-neutral plasma is confined within the ASB trap, the electric potential has an extremum near the trap's center, and the density and temperature tend to be lower near the trap's center than at the plasma's edge. The results of the simulations indicate that a three-dimensional electrostatic potential hill is formed by the space charge of a positron plasma confined by an ASB trap, and confinement of a second oppositely signed species, such as antiprotons, may be possible within the space charge of the positron plasma.

\section{ACKNOWLEDGMENTS}

The authors would like to thank Erin Thornton (UNT), Dr. Ryan Phillips (AFRL), and Dr. David Grote (LLNL) for helpful discussions regarding the Warp simulations. Computational resources were provided by UNT's High Performance Computing Services. This material is based upon work supported by the National Science Foundation under Grant Nos. PHY-1803047 and PHY-1500427 and by the Department of Energy under Grant No. DE-FG02-06ER54883. This endeavor was undertaken at the
University of North Texas and does not include any contribution or review by Ball Aerospace.

\section{REFERENCES}

'J. L. Pacheco, C. A. Ordonez, and D. L. Weathers, Phys. Plasmas 19, 102510 (2012).

${ }^{2}$ M. Ahmadi, B. X. R. Alves, C. J. Baker, W. Bertsche, A. Capra, C. Carruth, C. L. Cesar, M. Charlton, S. Cohen, R. Collister, S. Eriksson, A. Evans, N. Evetts, J. Fajans, T. Friesen, M. C. Fujiwara, D. R. Gill, J. S. Hangst, W. N. Hardy, M. E. Hayden, M. E. Hayden, C. A. Isaac, M. A. Johnson, S. A. Jones, S. Jonsell, L. Kurchaninov, N. Madsen, D. Maxwell, J. T. K. McKenna, S. Menary, J. M. Michan, T. Momose, J. J. Munich, K. Olchanski, A. Olin, P. Pusa, C. $\varnothing$. Rasmussen, F. Robicheaux, R. L. Sacramento, M. Sameed, E. Sarid, D. M. Silveira, D. M. Starko, G. Stutter, C. So, T. D. Tharp, R. I. Thompson, D. P. van der Werf, and J. S. Wurtele, Nature 561, 211 (2018).

${ }^{3}$ G. Gabrielse, R. Kalra, W. S. Kolthammer, R. McConnell, P. Richerme, D. Grzonka, W. Oelert, T. Sefzick, M. Zielinski, D. W. Fitzakerley, M. C. George, E. A. Hessels, C. H. Storry, M. Weel, A. Müllers, and J. Walz, Phys. Rev. Lett. 108, $113002(2012)$.

${ }^{4}$ N. Kuroda, S. Ulmer, D. J. Murtagh, S. Van Gorp, Y. Nagata, M. Diermaier, S. Federmann, M. Leali, C. Malbrunot, V. Mascagna, O. Massiczek, K. Michishio, T. Mizutani, A. Mohri, H. Nagahama, M. Ohtsuka, B. Radics, S. Sakurai, C. Sauerzopf, K. Suzuki, M. Tajima, H. A. Torii, L. Venturelli, B. Wünschek, J. Zmeskal, N. Zurlo, H. Higaki, Y. Kanai, E. Lodi Rizzini, Y. Nagashima, Y. Matsuda, E. Widmann, and Y. Yamazaki, Nat. Commun. 5, 3089 (2014).

${ }^{5}$ A. Kellerbauer, M. Amoretti, A. S. Belov, G. Bonomi, I. Boscolo, R. S. Brusa, M. Buchner, V. M. Byakov, L. Cabaret, C. Canali, C. Carraro, F. Castelli, S. Cialdi, M. de Combarieu, D. Comparat, G. Consolati, N. Djourelov, M. Doser, G. Drobychev, A. Dupasquier, G. Ferrari, P. Forget, L. Formaro, A. Gervasini, M. G. Giammarchi, S. N. Gninenko, G. Gribakin, S. D. Hogan, M. Jacquey, V. Lagomarsino, G. Manuzio, S. Mariazzi, V. A. Matveev, J. O. Meier, F. Merkt, P. Nedelec, M. K. Oberthaler, P. Pari, M. Prevedelli, F. Quasso, A. Rotondi, D. Sillou, S. V. Stepanov, H. H. Stroke, G. Testera, G. M. Tino, G. Trenec, A. Vairo, J. Vigue, H. Walters, U. Warring, S. Zavatarelli, and D. S. Zvezhinskij, Nucl. Instrum. Methods Phys. Res., Sect. B 266, 351 (2008).

${ }^{6}$ P. Indelicato, G. Chardin, P. Grandemange, D. Lunney, V. Manea, A. Badertscher, P. Crivelli, A. Curioni, A. Marchionni, B. Rossi, A. Rubbia, V. Nesvizhevsky, D. Brook-Roberge, P. Comini, P. Debu, P. Dupre, L. Liszkay, B. Mansoulie, P. Perez, J. M. Rey, B. Reymond, N. Ruiz, Y. Sacquin, B. Vallage, F. Biraben, P. Clade, A. Douillet, G. Dufour, S. Guellati, L. Hilico, A. Lambrecht, R. Guerout, J. P. Karr, F. Nez, S. Reynaud, C. I. Szabo, V. Q. Tran, J. Trapateau, A. Mohri, Y. Yamazaki, M. Charlton, S. Eriksson, N. Madsen, D. P. van der Werf, N. Kuroda, H. Torii, Y. Nagashima, F. Schmidt-Kaler, J. Walz, S. Wolf, P. A. Hervieux, G. Manfredi, A. Voronin, P. Froelich, S. Wronka, and M. Staszczak, Hyperfine Interact. 228, 141 (2014).

${ }^{7}$ M. E. Glinsky and T. M. O’Neil, Phys. Fluids B 3, 1279 (1991).

${ }^{8}$ F. Robicheaux, Phys. Rev. A 73, 033401 (2006).

${ }^{9}$ C. A. Ordonez, Phys. Rev. E 76, 017402 (2007)

${ }^{10} \mathrm{C}$. A. Ordonez and D. L. Weathers, Phys. Plasmas 15, 083504 (2008).

${ }^{11}$ C. A. Ordonez, J. Appl. Phys. 106, 024905 (2009).

${ }^{12}$ R. M. Hedlof and C. A. Ordonez, AIP Adv. 7, 115123 (2017).

${ }^{13}$ C. A. Ordonez, J. Appl. Phys. 104, 054903 (2008).

${ }^{14}$ C. A. Ordonez, Phys. Plasmas 15, 114507 (2008).

15. L. Pacheco, C. A. Ordonez, and D. L. Weathers, Nucl. Instrum. Methods Phys. Res., Sect. B 332, 312 (2014)

${ }_{17}^{16}$ R. M. Hedlof and C. A. Ordonez, Phys. Proc. 66, 95 (2015).

${ }^{17}$ T. J. Dolan, Plasma Phys. Controlled Fusion 36, 1539 (1994).

${ }^{18}$ A. Friedman, R. H. Cohen, D. P. Grote, S. M. Lund, W. M. Sharp, J. L. Vay, I. Haber, and R. A. Kishek, IEEE Trans. Plasma Sci. 42, 1321 (2014).

${ }^{19}$ R. M. Hedlof and C. A. Ordonez, AIP Conf. Proc. 1928, 020003 (2018)

${ }^{20}$ C. A. Ordonez, D. D. Dolliver, Y. Chang, and J. R. Correa, Phys. Plasmas $\mathbf{9}$, 3289 (2002)

${ }^{21}$ M. Horky, W. J. Miloch, and V. A. Delong, Phys. Rev. E 95, 043302 (2017). 